\title{
Impact of Caryolanemagnolol on Gait and Functional Mobility on Individuals with Huntington's Disease
}

\author{
Ran $\mathrm{Du}^{*}$ \\ Department of Neurology, The First Affiliated Hospital of Zhengzhou University, Zhengzhou 471000, China
}

*For correspondence: Email: duran19665@gmail.com; Tel/Fax: 0086-371-66913114

Received: 4 March 2015

Revised accepted: 30 July 2015

\begin{abstract}
Purpose: To highlight the impact of caryolanemagnolol on gait measures in forward walking, balance and mobility measures, as well as in hand and forearm function measures.

Methods: Twenty patients with Huntington's disease (HD) on stable doses of caryolanemagnolol were evaluated after medication and again following resumption of medication. The improvement in spatiotemporal gait parameters was measured by GAITRite, balance and/or mobility using Tinetti mobility test (TMT), five times sit-to-stand test (5TSST), and six-condition Romberg tests, as well as the function of the hand and forearm by finger tapping and Luria tests on Huntington's disease rating scale (UHDRS) motor scale.

Results: The results demonstrated significant improvement in the unified UHDRS motor scores, Tinetti mobility test (TMT) total and balance subscale scores, and the five times sit-to-stand test when oncaryolanemagnolol compared to off-caryolanemagnolol. Spatiotemporal gait measures, the six condition Romberg test, and UHDRS hand and forearm function items remained unaffected on caryolanemagnolol treatment. Improved TMT and 5TSST performance when on drug indicates that caryolanemagnolol use may improve balance and functional mobility in individuals with $H D$.

Conclusions: Caryolanemagnolol improves chorea and functional mobility by improving dynamic balance in individuals without any negative impact on motor function in walking and transfers.
\end{abstract}

Keywords: Huntington's disease, Caryolanemagnolol, Gait measures, Choreic movements, Mobility tests

Tropical Journal of Pharmaceutical Research is indexed by Science Citation Index (SciSearch), Scopus, International Pharmaceutical Abstract, Chemical Abstracts, Embase, Index Copernicus, EBSCO, African Index Medicus, JournalSeek, Journal Citation Reports/Science Edition, Directory of Open Access Journals (DOAJ), African Journal Online, Bioline International, Open-J-Gate and Pharmacy Abstracts

\section{INTRODUCTION}

One of the characteristic and dangerous symptoms associated with Huntington's disease (HD) is chorea. The increase in amplitude of choreic movements leads to a decrease in balance, walking, and daily activities like getting up and down from a chair and increases fall rates [1]. Treatment of chorea has been reported to improve motor function, safety, and quality of life. In a variety of movement disorders reduction of monoamines particularly dopamine has been used to treat involuntary movements in the brain.
In 2007, tetrabenazine, a vesicular monoamine transporter 2 inhibitor was approved by FDA for the treatment of chorea in HD.

Chorea can be decreased by the use of several neuroleptics and tetrabenazine. Administration of the neuroleptic haloperidol to individuals affected by $H D$ has no effects on spatial or temporal measures of gait $[2,3]$. However, the high doses of neuroleptics like olanzapine and zotepine have shown a significant improvement in chorea as well as fine motor tasks, oral functions, and some gait measures $[4,5]$. Use of tetrabenazine for 80 
weeks in individuals with HD led to improvement in chorea scores and in global improvement rating [6]. Tetrabenazine administration has been shown to improve the ability of lifting the large and light objects [7]. In another study tetrabenazine was shown to improve both chorea and functional measures of balance and gait like, Berg balance scale and dynamic gait index [8].

However, some of the patients have reported deterioration in gait or increased falls after tetrabenazine treatment. It is reported that older individuals are more likely to develop parkinsonian features on tetrabenazine treatment [9]. Thus the development of an alternative therapeutic strategy is highly needed to overcome this limitation.

The bark of Magnolia obovata has been used in China, Japan, and Korea as a traditional medicine. From the extract of this bark was isolated caryolanemagnolol (Figure 1) which showed neurotrophic activity [10,11]. The compound accelerated neurite sprouting and neuronal network formation $0.1 \mu \mathrm{M}$. The current study was designed to investigate the effect of caryolanemagnolol on spatiotemporal gait parameters, balance and/or mobility and function of the hand and forearm.

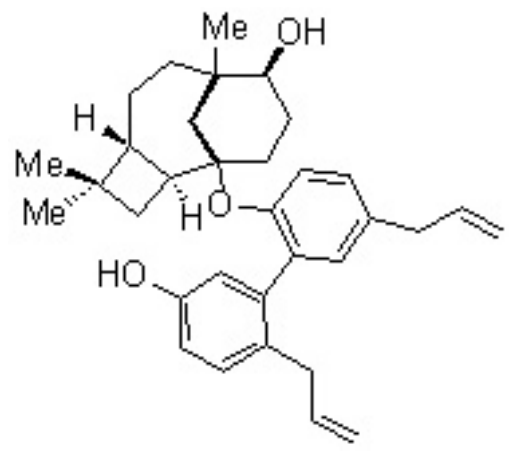

Figure 1: Structure of caryolanemagnolol

\section{EXPERIMENTAL}

All the subjects were required to attend the center for off-caryolanemagnolol for at least $12 \mathrm{~h}$ prior to undergoing study tests. Subjects arrived at the center for off-caryolanemagnolol and the demographic information was obtained. The subjects were then tested for the following measures: (a) UHDRS motor scale $(0-4)$ was used for motor symptoms. Total motor scores (range 0 - 124), total chorea scores (range 0 28 ), and total forearm and hand function scores (range 0 - 12) [12] were calculated for each subject. The higher scores indicate greater disability. (b) A 16' long GAITRite $®$ system (CIR
Systems, Inc, Haverton, PA) which calculates swing percent and double support percent for each leg was employed to measure spatiotemporal parameters of walking. GAITRite $\AA$ software program averaged the walks of the subjects for four separate passes across the GAITRite $\AA$ at their comfortable self-selected speed. GAITRite ${ }^{\circledR}$ measures are reliable and valid in individuals with HD [13]. (c) TMT, a reported method $[14,15]$ was used to measure balance, functional mobility, and fall risk. The highest scale is 28 (balance subscale $=16$, gait subscale $=12$, and the higher scores indicate better performance. (d) 5TSST measures the ability of the subject to balance in a standard armless chair while standing up and sitting down five times as quickly.

5TSST has been shown to be a reliable and valid measure in older adults and other patient populations [16]. (e) In Six Condition Romberg test of static balance subjects need to stand with arms crossed over the chest and perform each of the following for $30 \mathrm{~s}: 1$ ) feet together, 2) feet together and eyes closed, 3) feet aligned in a tandem heel-to-toe position, 4) tandem position and eyes closed, 5) tandem position while counting backwards by 3 's from 100, and 6) tandem position while counting backwards by 3 's from 100 with eyes closed [17].

After the initial testing, the subjects were instructed to take their usual dose of caryolanemagnolol. The same tests were then administered following a $2-3 \mathrm{~h}$ resting period. Kenney et al [18] documented that chorea scores begin to improve within an hour of administration of caryolanemagnolol, with a mean duration of effect on chorea lasting $5.4 \pm 1.3 \mathrm{~h}$ [18]. The majority of subjects had maximal benefit (physician rating) within $2 \mathrm{~h}$ of administration [18]. A neurologist with the European Huntington's Disease Network UHDRS motor rater certification administered the UHDRS motor scale. All other tests were administered by one of three physical therapist researchers who had considerable experience with test administration. The patients with a genetically confirmed diagnosis of HD, who had been maintained on a stable dose of caryolanemagnolol for at least 30 days, were selected for this study. The patients having another neurologic disorder (i.e., CVA, TBI), presence of an orthopedic condition that impeded the ability to walk and pregnancy were excluded from the study. A written informed consent, approved by the Institutional Review Board, was obtained from all patients confirming that they are aware of the nature of study performed. 
Each subject was evaluated by the same tester for off and on-caryolanemagnolol tests. All subjects had practice trials of each test prior to the recorded testing to ensure understanding and to eliminate learning effects. During all gait and functional tests, subjects were guarded by one of the investigators. Safety was ensured during the off-caryolanemagnolol time period by asking a family member to drive them to the testing site and by close monitoring by the investigators or family members. Previous studies have shown no significant adverse effects from the sudden withdrawal of caryolanemagnolol $[18,19]$.

\section{Statistical analysis}

SPSS, version 2 was used for statistical analyses and Shapiro-Wilk Test of Normality for normality of the obtained data. Wilcoxon matched-pairs signed-rank test was used for analysis of gait and functional data. The coefficient of variation (CV) values were calculated for step time, stride length, swing time and double support time. The fall risk in individuals with HD has been linked with increased variability of gait measures [1].

\section{RESULTS}

Among twenty three individuals enrolled for this study, three were excluded due to absence in follow-up testing $(n=2)$ and termination of the medication $(n=1)$. Demographic parameters for the twenty individuals who completed study was recorded (Table 1).

Out of twenty individuals 13 were men and 7 women with mean age of $49.5 \pm 8.2$ ranging from 30 - 64 years. The mean time since the symptom onset was $9.3 \pm 6.5$ years with an average of 3.1-19 years and the mean CAG repeat size was $39.8 \pm 3.2$ with range $38-53$. The chorea scores for all the subjects were more than 10 . The patients were administered $50.8 \pm$ $10.0 \mathrm{mg}$ caryolanemagnolol daily for one month. The results showed a significant improvement in mean UHDRS total motor scores. The UHDRS total motor scores recorded was $57.45 \pm 10.34$ $(29-76)$ and $44.25 \pm 10.72(23-65)(p=.001)$ off-and on-caryolanemagnolol, respectively. The chorea scores were $20.02 \pm 5.2(10-29)$ and $10.34 \pm 4.5(9-17)$ in the off- and oncaryolanemagnolol state, respectively. Treatment with caryolanemagnolol led to significant total motor scores even after excluding the chorea measures of the face, trunk, upper extremity and lower extremity. These scores were $38.18 \pm$ $11.03(19-61)$ and $34.82 \pm 11.50(17-58)$ offand on-caryolanemagnolol, respectively. The results revealed that there was no significant effect of caryolanemagnolol on spatiotemporal parameters of gait (Table 2).

There was a significant improvement in TMT total and balance subscale scores in all the patients on caryolanemagnolol. 5TSST scores for all the patients were also significantly better when oncaryolanemagnolol compared to offcaryolanemagnolol (Table 3). However, the Six Item Romberg total time remained unaffected on exposure to caryolanemagnolol. On the other hand, UHDRS forearm and hand function scores showed improvement. All the patients and their caregivers were satisfied with the treatment. The patients stated that they felt stable and balanced on caryolanemagnolol treatment. They stated that they felt their hand function improved while on caryolanemagnolol.

Table 1: Demographic parameters for the selected individuals

\begin{tabular}{ll}
\hline Variable & Value \\
\hline Sex (men, women) & 13,7 \\
Age (mean, average) & $49.5 \pm 8.2,30-64$ years \\
Symptom onset (mean, average) & $9.3 \pm 6.5,3.1-19$ years \\
CAG repeat size (mean, average) & $39.8 \pm 3.2,38-53$ \\
Chorea scores (mean) & $>10 \pm \mathrm{SD}$ \\
\hline
\end{tabular}

Table 2: Comparison of gait parameters on- and off-caryolanemagnolol

\begin{tabular}{|c|c|c|c|c|}
\hline \multirow{2}{*}{$\begin{array}{l}\text { Outcome measure } \\
(n=20)\end{array}$} & \multicolumn{2}{|c|}{ Mean \pm SD } & \multirow[t]{2}{*}{$\mathbf{W}$} & \multirow{2}{*}{$\begin{array}{c}\text { Sig. } \\
\text { (2-tailed) }\end{array}$} \\
\hline & Off & On & & \\
\hline Velocity $(\mathrm{m} / \mathrm{s})$ & $1.18 \pm 0.12$ & $1.23 \pm 0.19$ & 0.65 & 0.389 \\
\hline Stride length $(\mathrm{cm})$ & $126.87 \pm 26.23$ & $123.56 \pm 20.13$ & 0.22 & 0.795 \\
\hline Swing percent of gait cycle (\%) & $35.02 \pm 3.05$ & $35.98 \pm 3.78$ & -0.69 & 0.523 \\
\hline Double support percent of cycle (\%) & $26.45 \pm 5.09$ & $29.47 \pm 5.76$ & -0.98 & 0.312 \\
\hline Stride length CV & $7.43 \pm 5.32$ & $7.04 \pm 3.88$ & 0.59 & 0.645 \\
\hline Double support time CV & $12.63 \pm 5.41$ & $12.69 \pm 6.12$ & 0.57 & 0.489 \\
\hline
\end{tabular}


Table 3: Comparison of functional outcome measures on- and off-caryolanemagnolol

\begin{tabular}{lcccc}
\hline Outcome measure & \multicolumn{2}{c}{ Mean $\mathbf{E}$ SD } & W & Sig. \\
(n= 20) & Off & On & & (2-tailed) \\
\hline Tinetti Mobility Test (scores) & $16.32 \pm 5.12$ & $20.05 \pm 5.64$ & 5.34 & 0.003 \\
Tinetti Mobility Test Balance Subscale (scores) & $10.44 \pm 3.31$ & $14.16 \pm 3.42$ & -5.35 & 0.002 \\
Tinetti Mobility Test Gait Subscale (scores) & $6.25 \pm 3.67$ & $5.97 \pm 2.89$ & -2.18 & 0.109 \\
Five Times Sit-to-Stand Test (s) & $16.98 \pm 4.54$ & $10.78 \pm 4.12$ & 4.13 & 0.013 \\
Six Item Romberg Test (total time completed in s) & $69.87 \pm 18.34$ & $86.44 \pm 30.45$ & 2.27 & 0.098 \\
UHDRS forearm and hand function scores (items 6- & $14.11 \pm 5.78$ & $9.94 \pm 6.37$ & 1.98 & 0.057 \\
8) (scores) & & & \\
\hline
\end{tabular}

\section{DISCUSSION}

The results of our study demonstrate that caryolanemagnolol leads to improvements in balance and functional mobility in individuals with HD. Treatment with caryolanemagnolol did not exhibit parkinsonian gait changes in the patients. The improvements in the balance subscale rather than the gait subscale indicate significantly better total TMT score on-caryolanemagnolol treatment. The balance subscale includes observations of the individual getting up and returning back to sitting position and turning $360^{\circ}$. Caryolanemagnolol induced improvements in these activities have important clinical implications because patients with HD commonly suffer falls while standing up, sitting down and executing turns. We did not observe significant improvement in static standing balance under altered somatosensory conditions oncaryolanemagnolol treatment by using the six condition Romberg test state. Therefore, caryolanemagnolol has a greater impact on balance control systems that maintain balance during dynamic functional tasks compared to those that control balance during static tasks. The improved TMT scores observed in our study suggests that caryolanemagnolol use can improve performance on the Berg Balance Scale and the Dynamic Gait Index in individuals with HD.

Caryolanemagnolol treatment has no effect on the spatiotemporal gait parameters and CVs measured on the GAITRite®. It did not produce symptoms like bradykinesia and rigidity in any of the patients. There was no significant change in the UHDRS forearm and hand function scores between on-caryolanemagnolol and offcaryolanemagnolol. The mechanism behind the improvements in motor function by caryolanemagnolol is not fully clear. We are not certain whether these improvements are due to changes in severity of chorea, any other neurologic effect or due to combination of effects of caryolanemagnolol. A significant improvement in the total UHDRS motor scores oncaryolanemagnolol versus off-caryolanemagnolol was observed, even when the chorea scores were removed. The decrease observed in total UHDRS motor scores without chorea scores appears to be related to combined improvements in the hand and forearm function items and in the retropulsion pull test, gait, and tandem walking items, as none of these items were significantly different on-caryolanemagnolol and offcaryolanemagnolol when analyzed independently. The improvements in the pull test, gait, and tandem walking items are most likely related to improved dynamic balance as supported by our findings on the TMT.

\section{CONCLUSION}

Findings from this study demonstrate that caryolanemagnolol improves chorea and functional mobility by improving dynamic balance in individuals without any negative impact on motor function in walking and transfers.

\section{REFERENCES}

1. Grimbergen $Y A$, Knol MJ, Bloem BR, Kremmer BP, Roos RA, Munneke M. Falls and gait disturbances in Huntington's disease. Mov Disord 2008; 23: 970-976.

2. Koller WC, Trimble J. The gait abnormality of Huntington's disease. Neurology 1985; 35: 14501454.

3. Girotti F, Carella F, Scigliano G, GrassiMP, Soliveri $P$, Giovannini $P$, et al. Effect of neuroleptic treatment on involuntary movements and motor performances in Huntington's disease. J Neurol Neurosurg Psychiatry 1984; 47: 848-852.

4. Bonelli RM, Niederwieser G, Tribl GG, Koltringer P. Highdose olanzapine in Huntington's disease. Int Clin Psychopharmacol 2002; 17: 91-93.

5. Bonelli RM, Niederwieser G, Lahousen T, Hofmann $P$. Zotepine in Huntington's disease. Hum Psychopharmacol 2003; 18: 227-229.

6. Frank S. Tetrabenazine as anti-chorea therapy in Huntington disease: an open-label continuation study. Huntington Study Group/TETRA-HD Investigators. BMC Neurol 2009; 9: 62.

7. Ferrara J, Mostile G, Hunter C, Adam O, Jankovic J. Effect of tetrabenazine on motor function in patients with Huntington disease. Neurol Ther 2012; 1: 1-8. 
8. Fekete R, Davidson A, Jankovic J. Clinical assessment of the effect of tetrabenazine on functional scales in Huntington disease: a pilot open label study. Tremor Other Hyperkinet Mov (N Y) 2012:2 [epub 2012 Aug 6].

9. Paleacu D, Giladi N, Moore O, Stern A, Honigman S, Badarny S. Tetrabenazine treatment in movement disorders. Clin Neuropharmacol 2004; 27: 230-233.

10. Fukuyama Y, Otoshi Y, Miyoshi K, Nakamura K, Kodama $M$, Nagasawa, Hasegawa $T$, Okazaki H, Sugawara M. Tetrahedron 1992, 48, 377-392.

11. Fukuyama Y, Otoshi Y, Kodama M. Tet. Lett. 1990, 31, 4477-4480.

12. Rao AK, Gordon AM, Marder KS. Coordination of fingertip forces during precision grip in premanifest Huntington's disease. Mov Disord 2011; 26: 862-869.

13. Rao AK, Quinn L, Marder KS. Reliability of spatiotemporal gait outcome measures in Huntington's disease. Mov Disord 2005; 20: 1033 1037.

14. Kloos A, Kegelmeyer DA, Young G, Kostyk S. Fall risk assessment using the Tinetti Mobility Test in individuals with Huntington's disease. Mov Disord 2010; 25: 2838-2844.

15. Quinn L, Khalil H, Dawes H, Fritz NE, Kegelmeyer D, Kloos $A D$, et al. Reliability and minimal detectable change of physical performance measures in individuals with pre-manifest and manifest Huntington disease. Phys Ther 2013; 93: 942-956.

16. Whitney SL, Wrisley DM, Marchetti GF, Gee MA, Redfern MS, Furman JM. Clinical measurement of sit-to-stand performance in peoplewith balance disorders: validity of data for the Five-Times-Sit-to-Stand Test. Phys Ther 2005; 85: 1034-1045.

17. Khasnis A, Gokula RM. Romberg's test. J Postgrad Med 2003; 49: 169-172.

18. Kenney C, Hunter C, Davidson A, Jankovic J. Short-term effects of tetrabenazine on chorea associated with Huntington's disease. Mov Disord 2007; 22: 10-13.

19. Jankovic J. Treatment of hyperkinetic movement disorders with tetrabenazine: a double-blind crossover study. Ann Neurol 1982; 11: 41-47. 